\title{
Two Omaliine Species (Coleoptera: Staphylinidae) New to Korea
}

\author{
Tae-Kyu Kim, Kee-Jeong Ahn* \\ Department of Biology, Chungnam National University, Daejeon 305-764, Korea
}

\begin{abstract}
A taxonomic study of two omaliine genera Acruliopsis Zerche and Omalium Gravenhorst in Korea is presented. Two species (Acruliopsis ussuriensis Zerche and Omalium japonicum Sharp) are reported for the first time in Korea. Diagnoses of those two genera with redescriptions of the species are presented. Acruliopsis ussuriensis is distinguished by more or less short elytra, a pair of conspicuous processes on male sternite VII and emargination of outer lateral margin of paramere at base. Omalium japonicum is distinguished by brown elytra and structure of aedeagus with swollen median lobe and expanded paramere. Habitus photographs and illustrations of diagnostic characters are provided.
\end{abstract}

Keywords: Coleoptera, Staphylinidae, Omaliinae, Acruliopsis ussuriensis, Omalium japonicum, Korea

\section{NTRODUCTI ON}

The genera Acruliopsis Zerche and genus Omalium Gravenhorst contain four species (Zerche, 2003) and 141 species (Herman, 2001; Zerche, 2003) worldwide, respectively. All four Acruliopsis species (Zerche, 2003) and eight Omalium species (Smetana, 2004) are recorded in East Asia.

Little is known about the habitat and behavior of Acruliopsis species, however, the examined specimens were collected under bark of tree or by flight intercept trap (FIT). The Omalium species are found in various habitats such as decayed vegetable matters including seaweeds, manure, fungi and bird nests (Steel, 1970; Newton et al., 2000).

In this paper, we report Acruliopsis ussuriensis Zerche and Omalium japonicum Sharp for the first time in Korea, and provide the habitus photographs and redescriptions of the species with illustrations of diagnostic features. The specimens for this study are deposited in the Chungnam National University Insect Collection (CNUIC), Daejeon, Korea.

\section{RESULTS}

Order Coleoptera Linné, 1758

Family Staphylinidae Latreille, 1802

Subfamily Omaliinae MacLeay, 1825

Genus ${ }^{1 *}$ Acruliopsis Zerche, 2003

Acruliopsis Zerche, 2003: 299.

Type species: Acruliopsis watanabei Zerche.

Diagnosis. Body more or less convex and parallel-sided, covered with coarse punctures and pubescence; maxillary palpomere 4 almost as wide as and about three times longer than 3; antenna relatively short and gradually incrassate distally; eye large and protruded, ocular ridge surrounding eye; ocelli distinct and apart widely; gular sutures divergent posteriorly; pronotum convex medially and transverse, lateral margin crenulate; mesocoxal cavities separated; abdominal tergite VII with apical palisade fringe; sternite III with conspicuous intercoxal process, transverse carina present and anterior half beyond carina impressed (Zerche, 2003).

2*Acruliopsis ussuriensis Zerche, 2003 (Figs. 1A, 2)

Acruliopsis ussuriensis Zerche, 2003: 305.

Korean name: ${ }^{1 *}$ 톱니가슴네 눈반날개속(신칭), ${ }^{2 *}$ 톱니가슴네눈반날개 (신칭)

\footnotetext{
(C) This is an Open Access article distributed under the terms of the Creative Commons Attribution Non-Commercial License (http://creativecommons.org/ licenses/by-nc/3.0/) which permits unrestricted non-commercial use, distribution, and reproduction in any medium, provided the original work is properly cited.

pISSN 2234-6953 eISSN 2234-8190
}

*To whom correspondence should be addressed

Tel: 82-42-821-5492, Fax: 82-42-822-9690

E-mail: kjahn@cnu.ac.kr 
Material examined. Korea: $1 \sigma^{7}$, Gangwon Prov.: Inje-gun, Girin-myeon, Jindong-ri, Mt. Jeombongsan, 18 Apr 2007, Kim YH, under bark (CNUIC); $10^{7}$, Pyeongchang-gun, Jinbu-myeon, Dongsan-ri, Mt. Odaesan, Sangwonsa, 16 Aug15 Sep 2001, Park SJ, Shin CW, ex FIT (CNUIC).

Description. Body length 2.3-2.6 mm (head to abdominal end), body brown to dark reddish brown and lustrous, appendages yellow to reddish brown, anterior part of pronotum and humeral region of elytra weakly brighter. Head broad, pentagonal, punctate sparsely and coarsely, about 1.3 times wider than long; eye about 2.3 times longer than temple; distance between ocelli about 1.8 times wider than distance between outside of ocellus and inner margin of eye; antennae reaching to anterior third of elytra; basal five antennomeres lustrous and remaining lusterless; scape robust, about 1.8 times longer than wide; pedicel ovoid, about 1.4 times longer than wide, slightly shorter and narrower than scape; antennomere 3 slender, slightly dilated apically, 1.3 times longer than wide, slightly shorter and narrower than pedicel; 4-5 ovoid, almost same in length and shape each other, about 1.4 times longer than wide; 6 subglobular, slightly longer than wide; 7-9 subpentagonal, slightly getting shorter and wider distally, about 1.06-1.20 times wider than long; 10 trapezoidal, about 1.3 times wider than long, slightly shorter than 9; 11 about 1.3 times longer than wide. Pronotum (Fig. 2A) subhexagonal transversely, convex medially and deplanate laterally, covered with punctures much coarser than head; lateral margin sinuated with crenulation, widest near middle, 1.6 times wider than long, about 1.4 times wider and 1.2 times longer than head; anterior margin almost straight, posterior margin arcuate; anterior angles obtuse and posterior one more or less angulate; posterior third of deplanate area more or less depressed. Elytra more or less convex laterally, punctate coarsely and densely, about 1.3 times wider than long, about 1.2 times wider and 1.5 times longer than pronotum; posterior margin truncated, lateral margin crenulated excluding posterior fourth; prosternal process long and acute with short carina longitudinally; ventral surface of middle tibia with about five or six granules arranged; ventral surface of metatibia roundly and shallowly excavated in apical two third. Abdomen punctate finely and sparsely, surface with dense reticulate microsculpture; tergite IV with pair of transverse oval patches of wing-folding spicules.

Male. Abdominal tergite VIII (Fig. 2B) narrowed posteriorly with truncated but weakly emarginated posterior margin; pair of small and obtuse processes present on posterior third of sternite VI, projecting postero-ventrally (Fig. 2C); conspicuous and acute processes present just before poste-

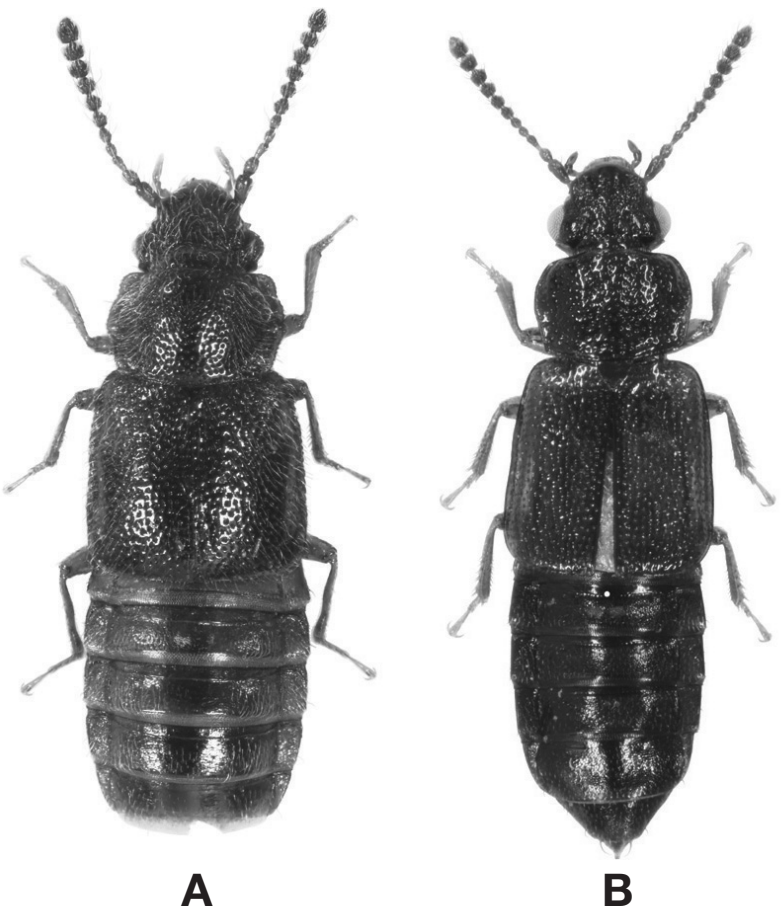

Fig. 1. Habitus photographs. A, Acruliopsis ussuriensis Zerche, male, $2.6 \mathrm{~mm}$; B, Omalium japonicum Sharp, male, $2.8 \mathrm{~mm}$.

rior margin of sternite VII, projecting postero-ventrally with apical setae, distance between them almost twice as wide as distance on sternite VI (Fig. 2C); sternite VIII (Fig. 2D) narrowed posteriorly, more or less extended triangularly. Aedeagus (Fig. 2E, F) trilobed; median lobe robust, gently narrowed apically with round apex; parameres little longer than median lobe, apical half of paramere constricted and curved inwardly, apical part expanded and inner surface excavated roundly.

Distribution. Korea, Russia (Far East).

${ }^{1 *}$ Genus Omalium Gravenhorst, 1802

Omalium Gravenhorst, 1802: 111; Hatch, 1957: 80; Zanetti, 1987: 205; Watanabe, 1990: 88; Downie and Arnett, 1996: 437; Newton et al., 2000: 337.

Type species: Staphylinus rivularis Paykull.

Diagnosis. Body more or less depressed, elongate and parallel-sided, covered with more or less coarse punctures; oblique short furrow or depression present in front of each ocellus; maxillary palpomere 4 almost as wide as and about two times longer than 3; mentum with pair of long setae on each side of posterior fourth; antennae gradually incrassate distally, antennomeres 8-10 transverse; pronotum with paired 


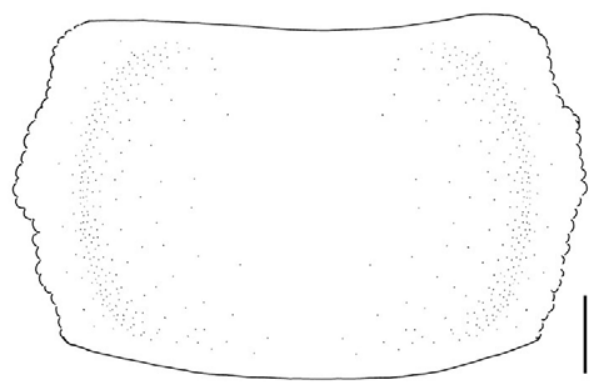

A

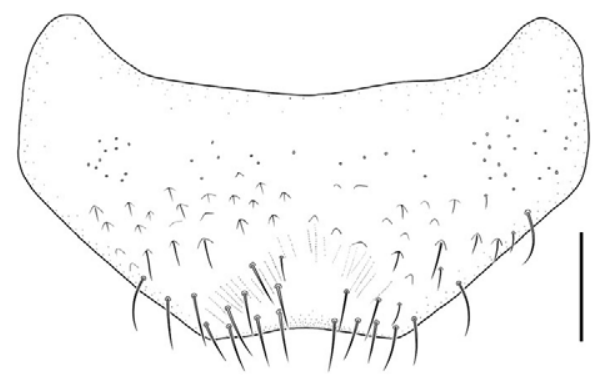

B

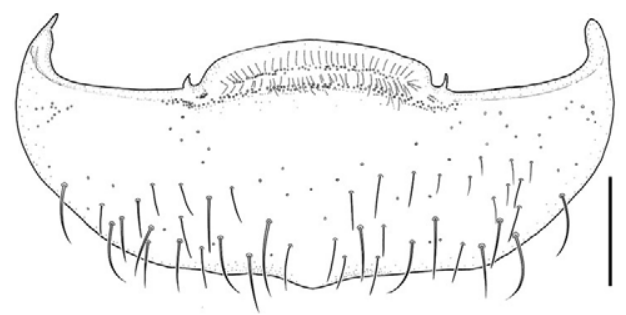

D

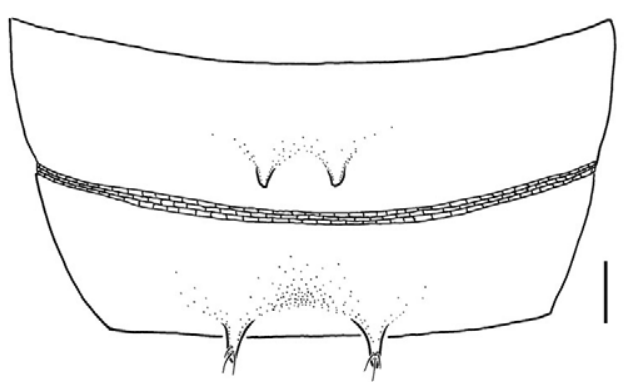

C

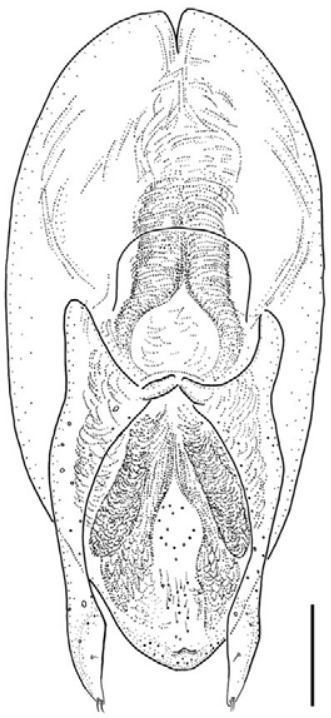

$\mathbf{E}$

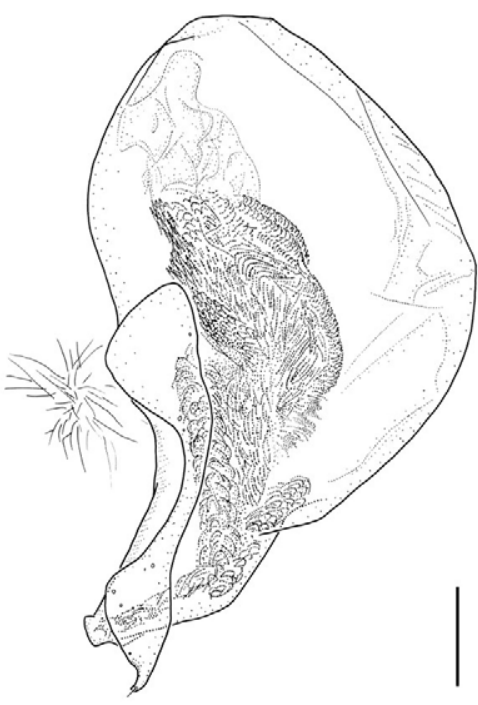

$\mathbf{F}$

Fig. 2. Acruliopsis ussuriensis. A, Pronotum, dorsal aspect; B, Male tergite VIII, dorsal aspect; C, Male sternites VI-VII, ventral aspect; D, Male sternite VIII, ventral aspect; E, Aedeagus, dorsal aspect; F, Aedeagus, lateral aspect. Scale bars: $A-F=0.1 \mathrm{~mm}$.

longitudinal depressions on posterior third; mesoventrite with longitudinal carina along midline; mesocoxal cavities separated; metatarsomere 5 longer than basal 4 combined; abdominal tergite VII with apical palisade fringe (Zanetti, 1987; Watanabe, 1990).

\section{1 * Omalium japonicum Sharp, 1874 (Figs. 1B, 3, 4)}

Omalium japonicum Sharp, 1874: 98; Nakane, 1971: 82; Watanabe, 1990: 95; 2013: 79; Shibata and Watanabe, 1999: 265; Herman, 2001: 517; Smetana, 2004: 263.

Material examined. Korea: $1 \sigma^{\nearrow}$, Gangwon Prov.: Samcheok-si, Wondeok-eup, Hosan-ri, 28 Apr 1985, Kim YS, under seaweed (CNUIC); 52 구38우 (in EtOH), Chungbuk Prov.: Yeongdong-gun, Hwanggan-myeon, $36^{\circ} 11^{\prime} 23.8^{\prime \prime} \mathrm{N}$, $127^{\circ} 53^{\prime} 44.3^{\prime \prime} \mathrm{E}, 248 \mathrm{~m}, 18$ May 2011, Kim TK, sifting, decaying food (CNUIC); 6 지 1 우 (1 지 1 우 on slides; 4 지 in EtOH), Chungnam Prov.: Gongju-si, Banpo-myeon, Mt. Gyeryongsan, $36^{\circ} 21^{\prime} 17.5^{\prime \prime} \mathrm{N}, 127^{\circ} 14^{\prime} 55.6^{\prime \prime} \mathrm{E}, 26$ Apr 2005 ,

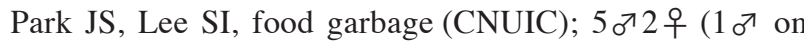
slide; $4 \sigma^{\top} 2$ 우 in EtOH), Gongju-si, Janggi-myeon, $36^{\circ} 29^{\prime}$ $25.1^{\prime \prime} \mathrm{N}, 127^{\circ} 14^{\prime} 11.3^{\prime \prime} \mathrm{E}, 70 \mathrm{~m}, 8$ May 2010, Lee SG, Jeon JH, food garbage (CNUIC); 3 우 (1우 on slide; 2 우 in EtOH), Seosan-si, Unsan-myeon, Yonghyeon Valley, 36 $45^{\prime} 5.7^{\prime \prime} \mathrm{N}$, $126^{\circ} 36^{\prime} 28^{\prime \prime} \mathrm{E}, 170$ m, 16 Apr 2009, Kim YH, Song JH, fungus on log and leaf litter (CNUIC); $2 \sigma^{\nearrow}$ (in EtOH), Unsan-

Korean name: ${ }^{1 *}$ 네 눈반날개 (신칭) 


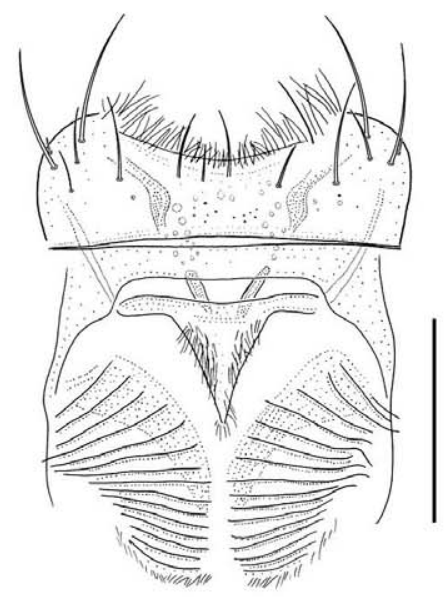

A

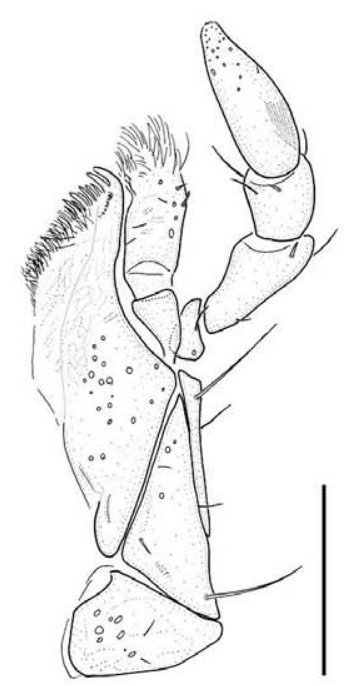

B
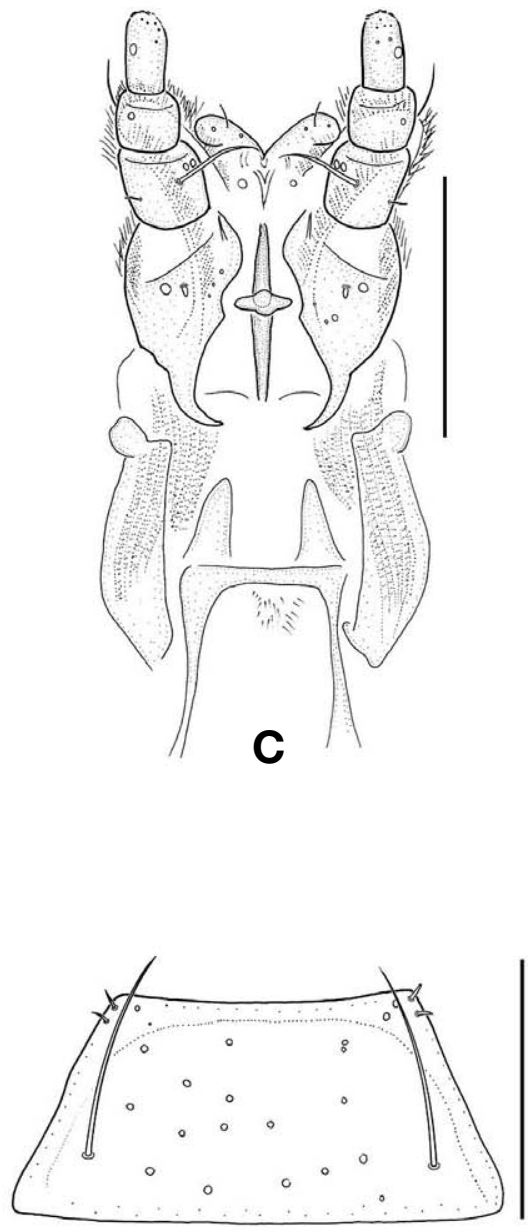

D

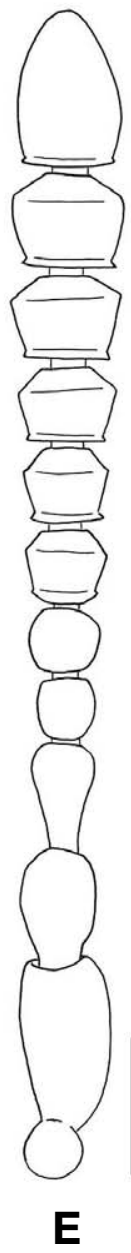

$\mathbf{E}$
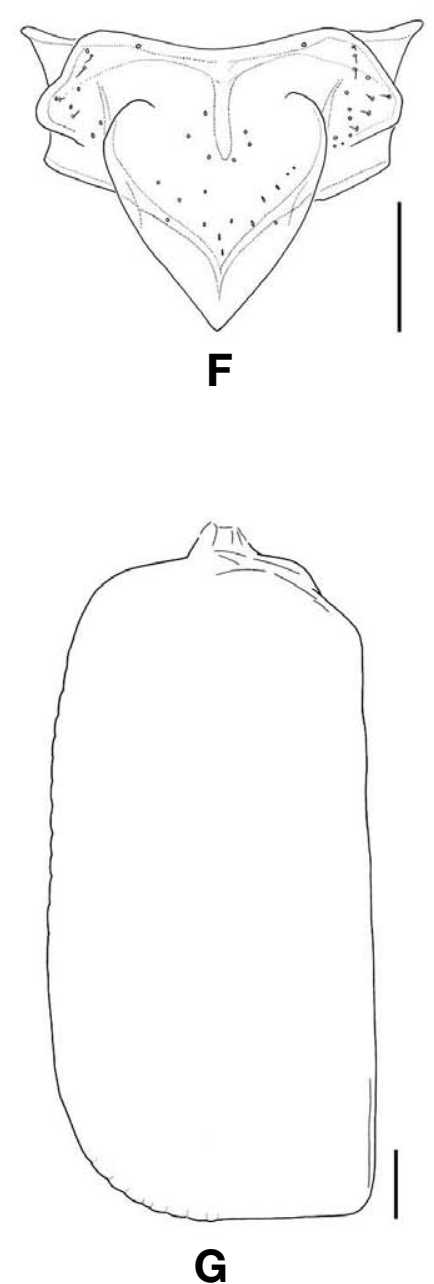

Fig. 3. Omalium japonicum. A, Labrum, dorsal aspect; B, Maxilla, ventral aspect; C, Labium, ventral aspect; $D$, Mentum, ventral aspect; E, Antenna; F, Scutellum of mesothorax, dorsal aspect; G, Elytron, dorsal aspect. Scale bars: $A-G=0.1 \mathrm{~mm}$.

myeon, Mt. Illaksan, $36^{\circ} 44^{\prime} 29.2^{\prime \prime} \mathrm{N}, 126^{\circ} 35^{\prime} 6.3^{\prime \prime} \mathrm{E}, 123 \mathrm{~m}$, 16 Apr 2009, Song JH, Lee SG, carrion (CNUIC); $10^{73} 3$ 우 (1 $\sigma^{\top}$ on slide; 3 우 in EtOH), Jeonbuk Prov.: Gochang-gun, Haeri-myeon, Goseong-ri, $35^{\circ} 26^{\prime} 40.7^{\prime \prime} \mathrm{N}, 126^{\circ} 33^{\prime} 0.6^{\prime \prime} \mathrm{E}, 1$ May 2007, Kim TK, Kim YH, under manure (CNUIC); 1 우, Jeonnam Prov.: Gurye-gun, Mt. Jirisan, Piagol, 24-26 May 2000, Choi HK, Song JH, Kim MS, ex bait trap (CNUIC); $10^{7}$ (in EtOH), Yeonggwang-gun, Baeksu-eup, Hasa-ri, $35^{\circ} 15^{\prime}$ 46.4' $\mathrm{N}, 126^{\circ} 21^{\prime} 22.1^{\prime \prime} \mathrm{E}, 15 \mathrm{~m}, 2$ May 2007, Kim TK, under manure (CNUIC); 1 우 (in EtOH), Hasa-ri, shell heap (CNUIC); $1 \mathrm{o}^{72}$ 우1?, Gyeongbuk Prov.: Ulsan-si, Mt. Kaji-san, 10-15 May 1999, Cho YB. ex bait trap (CNUIC); $1 \sigma^{7}$, Gyeongnam Prov.: Jinju-si, Gajoa-dong, Gyeongsang Univ., 13 Apr 2003, Lim CS, ex bait (CNUIC); $2 \sigma^{7}$, Gyeongsang Univ., 27 Apr
2003, Lim CS, ex bait (CNUIC); $10^{7}$, Gyeongsang Univ., 3 May 2003, Lim CS, ex bait (CNUIC); 1 우, Gyeongsang Univ., 19 Sep 2003, Lim CS, ex bait (CNUIC); 1 우, Sachoen, Guam, 5 May 1986, Lee KS, decayed fish (CNUIC); 1 우, Jeju Prov.: Namjeju-gun, Pyoseon-myeon, Gasi-ri, 18 Apr 1985, Lee KS (CNUIC); $10^{7} 1$ 우, Gasi-ri, 18 Apr 1985, Lee KS, fish (CNUIC); 1 우, Jeju-si, Jocheon-eup, Seongpanak, 15 May 1985, Lee KS, litter (CNUIC); 1 우, Seoguipo-si, Dongheungdong, Jeongbang-pokpo, 29 Oct 1985, Lee KS, decayed vege-

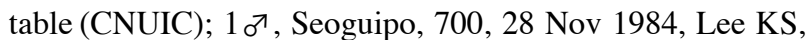
rotten wood (CNUIC); 1우, Seoguipo, 29 Nov 1984, Lee KS, cow dung (CNUIC); 1 ऽ$^{\top}$, Seoguipo, 2 Apr 1986, KS Lee, straw (CNUIC).

Description. Body length 2.7-3.2 mm (head to abdominal 


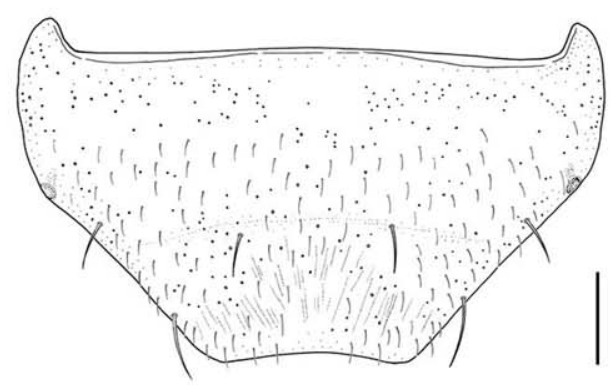

A

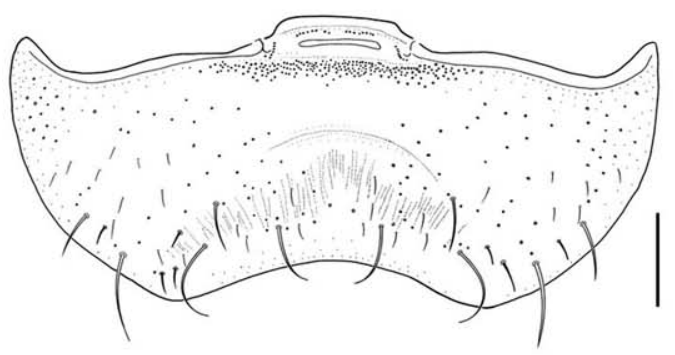

B

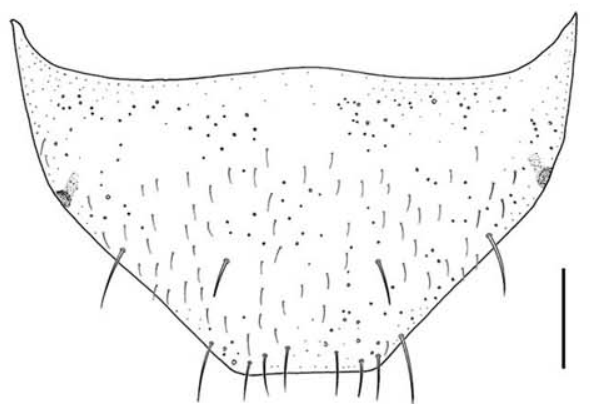

E

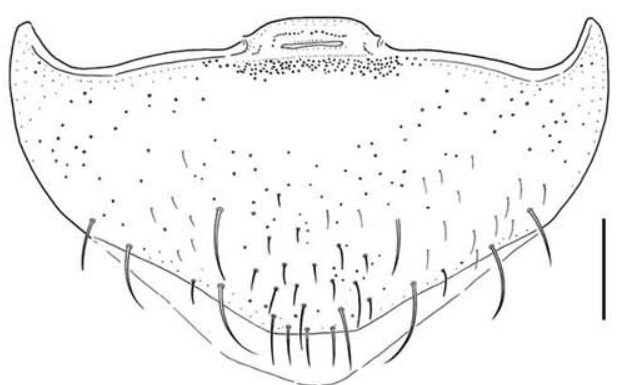

F

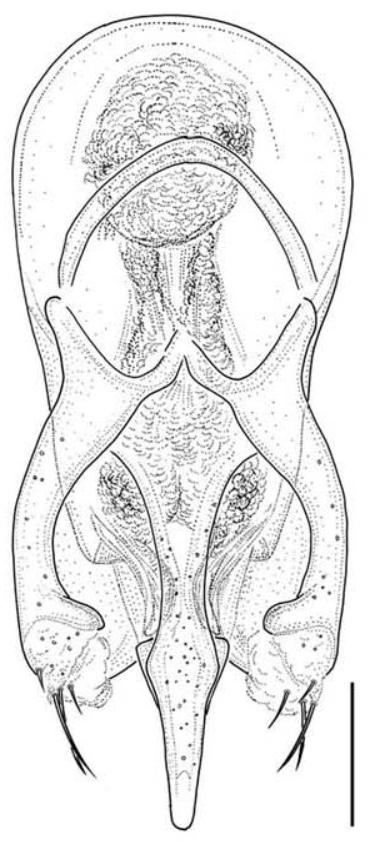

C

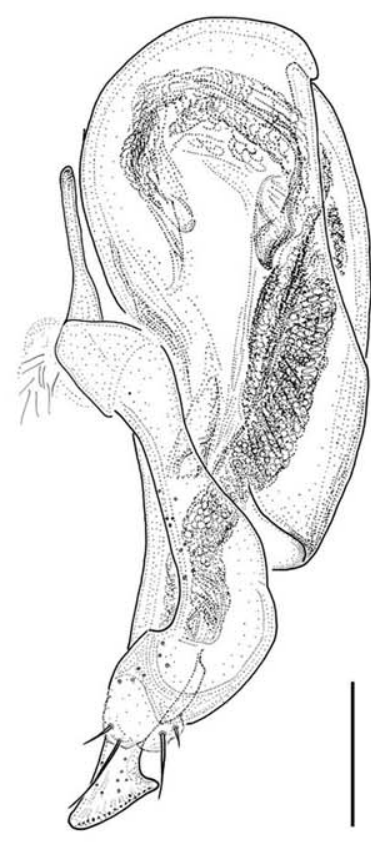

D
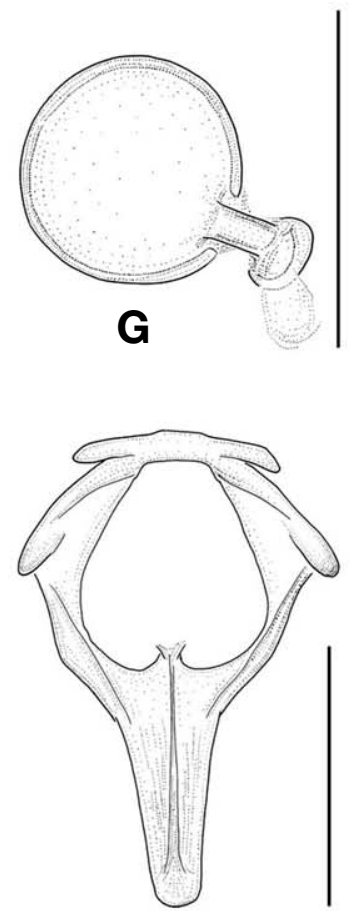

H

Fig. 4. Omalium japonicum. A, Male tergite VIII, dorsal aspect; B, Male sternite VIII, ventral aspect; C, Aedeagus, dorsal aspect; D, Aedeagus, lateral aspect; E, Female tergite VIII, dorsal aspect; F, Female sternite VIII, ventral aspect; G, Spermatheca; H, Accessary sclerite, dorsal aspect. Scale bars: $\mathrm{A}-\mathrm{H}=0.1 \mathrm{~mm}$. 
end), body dark brown, elytra and appendages yellowish brown to reddish brown. Head subpentagonal, covered with scattered coarse punctures, about 1.4 times wider than long; eye about 3.8 times longer than temple; distance between outside of ocellus and inner margin of eye about 1.3 times wider than distance between ocelli; antennae (Fig. 3E) reaching to posterior margin of pronotum, basal five antennomeres lustrous and remaining lusterless; scape robust, about 1.8 times longer than wide; pedicel ovoid, about 1.4 times longer than wide, relatively shorter and slightly narrower than scape; antennomere 3 more or less slender, slightly dilated apically, about 1.6 times longer than wide, slightly longer and narrower than pedicel; 4-5 subglobular, almost as wide as long; 6-10 subpentagonal and transverse, getting larger distally; 6-7 about 1.1 times wider than long; 8-10 about 1.2 times wider than long; 11 about 1.5 times longer than wide. Pronotum subquadrate transversely; lateral margin arcuate, widest anterior third, about 1.4 times wider than long, 1.3 times wider and longer than head; anterior margin almost straight, posterior margin arcuate; anterior angles subround and posterior one more or less angulate. Scutellum (Fig. 3F) heart-shaped, covered with scattered punctures and indistinct microsculpture. Elytra (Fig. 3G) covered with punctures and pubescence, lateral margin straight and almost parallel-sided, posterior margin truncated, slightly wider than long, 1.2 times wider and 1.6 times longer than pronotum; all tarsi with basal 4 combined shorter than tarsomere 5, metatarsomere 5 about 1.4 times longer than basal 4 combined. Abdomen subparallel; pair of suboval patches of wing folding spicules present on tergite IV.

Male. Protarsus more or less wide; abodominal tergite VIII (Fig. 4A) narrowed posteriorly, posterior margin weakly emarginate; sternite VIII (Fig. 4B) narrowed posteriorly, posterior margin broadly emarginate. Aedeagus (Fig. 4C, D) trilobed and symmetrical, median lobe more or less slender, swollen around middle; parameres shorter than median lobe, curved inwardly, lateral part rolling, apical part membranous with four apical setae.

Female. Abdominal tergite VIII (Fig. 4E) narrowed posteriorly with truncated posterior margin; sternite VIII (Fig. 4F) narrowed posteriorly with truncated posterior margin. Spermatheca (Fig. 4G) subglobular with attached tubule; accessory sclerite (Fig. $4 \mathrm{H}$ ) widest at anterior third and narrowed posteriorly with blunt apex, medial part opened like inverted heart-shape, posterior half carinate longitudinally.

Distribution. Korea, Japan.

\section{ACKNOWLEDGMENTS}

Financial support was provided by the National Institute of
Biological Resources (NIBR, Incheon) of Ministry of Environment, KOREA (1834-302) and the research on the species newly discovered in Korea was supported by a grant from the National Institute of Biological Resources (NIBR), funded by the Ministry of Environment (MOE) of the Republic of Korea (NIBR No. 2014-02-001).

\section{REFERENCES}

Downie NM, Arnett RH Jr, 1996. The beetles of Northeastern North America. Vol. 1. Introduction; suborders Archostemata, Adephaga, and Polyphaga, thru superfamily Cantharoidea. Sandhall Crane Press, Gainesville, pp. 437-438.

Gravenhorst JLC, 1802. Coleoptera Microptera Brunsvicensia nec non exoticorum quotquot exstant in collectionibus entomologorum Brunsvicensium in genera familias et species distribuit. Carolus Reichard, Braunschweig, pp. 111-119.

Hatch MH, 1957. The beetles of the Pacific Northwest. Part II: Staphyliniformia. University of Washington Press, Seattle, WA, pp. 80-81.

Herman LH, 2001. Catalog of the Staphylinidae (Insecta: Coleoptera). 1758 to the end of the second millennium. Part I. Introduction, history, biographical sketches, and omaliine group. Bulletin of the American Museum of Natural History, 265:1-650.

Latreille PA, 1802. Histoire naturelle, générale et particulière des crustacés et des insects. Tome troisième. Familles naturelles et genres. F. Dufart, Paris, pp. 13-468.

Linné, C. von, 1758. Systema naturae per regna tria narurae, secundum classes, ordines, genera, species, cum characteribus, differentiis, synonymis, locis. Editio duodecima, reformata. Tomus I. Laurentii Salvii, Stockholm, pp. 1-824.

MacLeay WS, 1825. Annulosa javanica, an attempt to illustrate the natural affinities and analogies of the insects collected in Java by Thomas Horsfield, M.D.F.L. \& G.S. and deposited by him in the Museum of the Honourable East-India Company. Kingsbury, Parbury, and Allen, London, pp. 1-50.

Nakane T, 1971. Staphylinidae. In: Iconographia insectorum Japonicorum, colore naturali edita, volumen II (Coleoptera). 4th ed. (Eds., Nakane T, Ohbayashi K, Nomura S, Kurosawa Y). Hokuryukan, Tokyo, pp. 81-100.

Newton AF, Thayer MK, Ashe JS, Chandler DS, 2000. 22. Staphylinidae. In: American beetles. Vol. 1. Archostemata, Myxophaga, Adephaga, Polyphaga: Staphyliniformia(Eds., Arnett RH, Thomas MC). CRC Press, Boca Raton, FL, pp. 272418.

Sharp D, 1874. The Staphylinidae of Japan. Transactions of the Entomological Society of London, 1874:1-103.

Shibata Y, Watanabe Y, 1999. Staphylinidae. In: The Coleoptera of Japan in color. Vol. II. 4th ed. (Eds., Uéno SI, Kurosawa Y, Satô M). Hoikusha Publishing, Higashiosaka, pp. 261321.

Smetana A, 2004. Staphylinidae, subfamily Omaliinae. In: Catalogue of Palaearctic Coleoptera, Volume 2, Hydrophiloidea- 
Histeroidea-Staphylinoidea (Eds., Löbl I, Smetana A). Apollo Books, Stenstrup, pp. 237-268.

Steel WO, 1970. The larvae of the genera of the Omaliinae (Coleoptera: Staphylinidae) with particular reference to the British fauna. Transactions of the Royal Entomological Society of London, 122:1-47.

Watanabe Y, 1990. A taxonomic study on the subfamily Omaliinae from Japan (Coleoptera, Staphylinidae). Memoirs of the Tokyo University of Agriculture, 31:55-391.

Watanabe Y, 2013. Subfamily Omaliinae MacLeay, 1825. In: Catalogue of Japanese Staphylinidae (Insecta: Coleoptera) (Eds., Shibata, Y., Maruyama, M). Bulletin of the Kyushu
University Museum, 11:73-80.

Zanetti A, 1987. Fauna d'Italia, Vol. XXV, Coleoptera, Staphylinidae, Omaliinae. Edizioni Calderini, Bologna, pp. 205240.

Zerche L, 2003. Acruliopsis gen. n. aus der Ostpaläarktis (Coleoptera, Staphylinidae, Omaliinae, Omaliini) (Beiträge zur Kenntnis ostpaläarktischer Insekten; 14). Beiträge zur Entomologie, 53:297-316.

Received February 3, 2014 Revised March 3, 2014 Accepted March 3, 2014 